\title{
Withania somnifera as an antistress herb
}

\author{
R Doomra $^{1}$, A Goyal $^{2}$ \\ ${ }^{1}$ HOD, Department of Pharmacology, Manav Rachna Dental College, Sector-43, Delhi-Surajkund Road, \\ Faridabad, Haryana, India \\ ${ }^{2}$ HOD, Department of Biochemistry, Manav Rachna Dental College, Sector-43, Delhi-Surajkund Road, \\ Faridabad, Haryana, India
}

Received: 10-08-2019 / Revised: 29-8-2019 / Accepted: 25-09-2019

\begin{abstract}
Stress, anxiety and depression have become common terms and are being used by todays' society so frequently that it appears as though nearly everyone has been affected by the same in some or the other way, irrespective of the age, sex, occupation or socio-economic status. The management of stress and anxiety therefore has become one of the important concerns, as finding peace and happiness is what all are striving for in this stressed-out era, and wherever people see a ray of hope to alleviate stress, the society is ready to go for the same. Stress is the cause of various illnesses ranging from psychiatric to chronic diseases like hypertension and diabetes mellitus. Studies have shown that some ayurvedic herbs act like adaptogens which increase resistance during stress. The adaptogens improve the response to stress and helps an individual to adapt by maintaining internal homeostasis in stressful situation. Withania somnifera, also known as Ashwagandha has been mentioned in Ayurveda and indigenous medical system for over 3000 years. It is also known as the Indian ginseng, as it is comparable to Panax Ginseng as an anti-stress herb.
\end{abstract}

Key words: Withania somnifera, stress, anxiety, adaptogen, Ashwagandha.

(C) The Author(s). 2019 Open Access. This work is licensed under a Creative Commons Attribution. The full terms of this license are available at our website and incorporate the Creative Commons Attribution. https://creativecommons.org/licenses/by/4.0/

\section{INTRODUCTION}

Ashwagandha, known as Withania somnifera, is also referred to as the 'royal herb', as it influences multiple functions in the body. Also known as the 'Indian ginseng', it belongs to the Solanaceae family. In Ayurveda, Withania somnifera is also known as 'rasayana' as it rejuvenates both mental and physical health.[1] Withania somnifera has anti-inflammatory, antimicrobial, immunemodulating, sedative, anti-tumour, neuroprotective and cardioprotective properties. This review is limited to the published articles in English language available on internet. Animal experiments and clinical trials have demonstrated the therapeutic use of Ashwagandha for anxiety, neurological disorders and Parkinson's disease. Ashwagandha has chemo-preventive properties, which are of benefit in patients undergoing radiation and chemotherapy. P Additionally, it has also demonstrated the ability to decrease reactive oxygen species and as an

*Correspondence

\section{R. Doomra}

HOD, Department of Pharmacology, Manav Rachna Dental College, Sector-43, Delhi-Surajkund Road, Faridabad, Haryana,India

E-mail: reena.mrdc@mrei.ac.in adaptogen for patients with nervous exhaustion, insomnia and fatigue due to stress.Prolonged exposure to stress can affect the physical and mental health of a person, which are responsible for fatigue, depression, and systemic ailments like hypertension, hyperglycaemia, peptic ulcer and immunosuppression.[2]

\section{Withania somnifera}

The biologically active chemical constituents are steroidal lactones (withanolides, withaferins), alkaloids and saponins. Withanolides have a steroidal nucleus with a side chain and six membered lactone ring. Withaferin A, $\mathrm{D}$ and Withanolide $\mathrm{G}$ are the main withanolides having pharmacologic activity.[3] The adaptogens help an individual to adapt to stressful situations, and Withania somnifera has several properties like adaptogens [4] and immunomodulatory action [5]. Withania somnifera enhances type-I immune response and cytokine production, and modulating the acute reactants. Alcoholic extract of Withania somnifera(roots and seeds) dissolved in normal saline was administered to mice $(100 \mathrm{mg} / \mathrm{kg}$ intraperitoneal, single dose) in a swimming performance test in water at $28^{\prime}-30^{\prime} \mathrm{C}$. It was observed that the Withania somnifera group showed an increase in the swimming time when compared to control, which was nearly double. Withania somnifera induced a state of 
nonspecific increase in resistance in a stressful situation.[6]In a randomized double-blind placebocontrolled study in patients having depression and anxiety as symptoms in schizophrenia were included. Withania somnifera extract was given to 66 patients for 12 weeks, and Withania somnifera showed significant improvement in depression and anxiety symptoms in these patients.[7]

\section{Stress and Withania somnifera}

The society has become complex, and there is everincreasing demand on an individual to cope with stress. Failure of successful adaptation to stress in an individual can lead to various mental and physical problems in an individual. Stress can be acute or chronic, and failure to adapt to a stressful situation can result in a number of disease conditions which affect most of the body systems. Acute stress leads to sympathetic nervous system overactivity manifesting as tachycardia and rise in arterial blood pressure, gluconeogenesis and hepatic glucose secretion. These changes lead to the elevation of catecholamines in the body.[8] If the stressful situation persists or an individual is not able to adapt physically or mentally, then the complex interplay of neuro-hormonal factors occurs, leading to release of cortisol in blood.[9] There are various attempts being made to handle stress in day-to-day situations, like yoga, breathing exercises, anxiolytic drugs like benzodiazepines. Withania somnifera has been shown to have adaptogenic activity, which helps the individual to adapt during the stress phase, thereby protects the individual from long-term stress. Withania somnifera promotes sound sleep due to alkaloids and glycosides which affect and nervous and immune system.[4,10]In a double-blind randomized placebo-controlled trial, 64 subjects with chronic stress were taken, and the study was done for 60 days. It was observed that Ashwagandha root extract (300 mg capsule) exhibited a highly significant reduction in perceived stress score, general health questionnaire-28, depression-anxiety stress scale data and serum cortisol levels.[11] Withania somnifera root extract (methanolic extract) has been found to be effective in stress-induced gastric ulcer in rats. The ulcers were induced by the indomethacin and swim stress treatment. It significantly reduced the ulcer index, gastric secretion and acidity. [12]Various studies have demonstrated the use of Ashwagandha for anxiety, neurological disorders, and insomnia. Chronic stress induced immunosuppression and depression were decreased by Ashwagandha. Studies have also demonstrated that Withania somnifera having GABA mimetic action.[13,14]]Long term effects of chronic stress may be related to increased levels of cortisol. Withania somnifera has shown promising results in decreasing anxiety and stress, and thereby promoting better adaptability to a stressful situation. However, more clinical trials are required to study the mechanism of action. The studies show promising results as mentioned in Ayurveda.

\section{Conflict of Interest: None; Source of Support: Nil}

\section{REFERENCES}

1. MA Weiner, $\mathrm{J}$ Weiner. Ashwagandha (Indian ginseng). Herbs that heal. Mill Vallery CA: Quantum Books; 1994. P. 70-2.

2. Suresha. S, Jayashankar.m and Vinu AK. Medicinal plants diversity in Muthathi Wild Life Sanctuary, Karnataka India. Indian J. Pharm. Biol. Res.2018; 6(3):13-22.

3. GR Elliot, C Eisdorfer. Stress and human health. New York; Springer Publishing 1982.

4. SK Verma and A Kumar. Therapeutic uses of Withania somnifera (ashwagandha) with a note on withanolides and its pharmacological actions. Asian J. Pharmaceutical and clinical research. 2011;4(Supp.1):1-4.

5. H Wagner, H Norr, $\mathrm{H}$ Winterhoff. Plant adaptogens. Phytomedicine. 1994; (1):63-78.

6. S Ghosal, RS Srivastava, SK Bhattacharya, SN Upadhayay, AK Jaiswal, U Chattopadhayay. Immunomodulatory and CNS effects of sitoindosides IX AND X, two glycowithanolides from Withania somnifera. Phytotherapy Research 1989:2;201-6.

7. LC Mishra, BB Singh, B Simon Degenais, LACC. Scientific basis for the therapeutic use of Withania somnifera (Ashwagandha) a review. Altern. Med. Review 2000; 5(4):334-3464.

8. JM Ganon, J Brar, A Rai, KNR Chengappa. Effects of standardized extract of Withania somnifera (Ashwagandha) on depression and anxiety symptoms in persons with schizophrenia participating in a randomized, placebo-controlled clinical trial. Annals Clin Psychiatry 2019; 31(2):123-129.

9. GP Chrousus. Stress and disorders of the stress system. Nature Reviews Endocrinology. 2009;5: 37481.

10. GP Chrousus, PW Gold. The concept of stress and stress system disorders. JAMA. 1992; 267:1244-52.

11. MB Modi, S Donga, Laxmipriya Del. Clinical evaluation of Ashokarishta, Ashwagandha Churna and Praval Pishti in the management of Menopausal syndrome. AYU Journal, 2012;33( 4):511-516.

12. $\mathrm{K}$ Chandrasekar, JKapoor, $S$ Anishetty. A prospective, randomized double-blind, placebocontrolled study of safety and efficacy of highconcentration full-spectrum extract of Ashwagandha root in stress and anxiety in adults. Indian $\mathrm{J}$ Psycol Med 2012,34(3): 255-62.

13. M Bhatnagar, SS Sisodia, $R$ Bhatnagar. Antiulcer and Antioxidant Activity of Asparagus racemosus WILLD and Withania somnifera DUNAL in Rats. Ann NY Acad Sci 2005; 1056:261-78.

14. GSingh, PKSharma, RDudhe, SSingh. Biological activities of Withania somnifera. Annuals of Biological Research, 2010;1(3):56-63. 